\title{
UN ESTUDIO EMPÍRICO SOBRE LA INVESTIGACIÓN EN EL DERECHO Y SU ENEMISTAD CON LA METODOLOGÍA CIENTÍFICA
}

Wilde Mollan*

Recibido: 07.07.2015

Aprobado: 30.09 .2015

\section{RESUMEN}

Las investigaciones de postgrado en derecho siguen en cuanto a su forma el enfoque de investigación cuantitativa. Hemos accedido a las tesis producto de esas investigaciones para observar si se ajustan a las pautas metodológicas mínimas exigidas en ciencias sociales.

\section{ABSTRACT}

Research postgraduate law follow as to form the focus of quantitative research. We have accessed to the thesis, the product of such research, to see if they meet the minimum methodological standards required in social sciences.

\section{PALABRAS CLAVE}

Derecho. Doctrina jurídica. Metodología. Investigación científica.

\section{KEY WORDS}

Law. Juridical doctrine. Methodology. Scientific investigation.

\section{Resultados}

De las tesis observadas $(n=$ 136), casi la mitad (41.18\%) no explicitan, establecen o determinan las variables de investigación, y el otro considerable número de tesis sí las explicitan estableciendo entre ellas exclusivamente relaciones de causalidad (56.62\%). Significativamente, las tres cuartas partes $(85.29 \%)$ del total de las tesis no operacionalizan las variables, la mitad de ese porcentaje es atribuido a la consecuencia natural de no explicitar previamente las variables, y sólo un reducido porcentaje (13.24\%) operacionalizan las variables relacionadas causalmente. El resultado más significativo es que más de la mitad de los trabajos de investigación (56.62 \%) no utilizan instrumentos para medir las variables por no estar determinadas, y el otro gran porcentaje (29.41 $\%)$ utiliza instrumentos de me- dición sin haber operacionalizado las variables a medir, lo que lleva a la utilización inapropiada de los diversos instrumentos aplicados.

\section{Conclusión}

Las investigaciones en derecho pretenden seguir el enfoque cuantitativo de investigación pero no se ajustan a sus exigencias mínimas metodológicas ampliamente aceptadas en las ciencias sociales.

\footnotetext{
* Investigador de Cienciax. Abogado socio del estudio jurídico Pro lus Abogados. Asesor externo en metodología de investigación en derecho de pregrado y postgrado. E-mail: wmlln@tuta.io.
} 


\section{INTRODUCCIÓN}

En muchas ocasiones he sido testigo de la desesperación que genera en las personas las indagaciones metódicas en derecho, más aún cuando son supervisadas por alguna institución superior que exige para realizarlas la adecuación a la metodología científica.

Me preguntaba, en un momento, al haberme detenido a revisar detalladamente algunas de estas indagaciones plasmadas en las tesis, si los investigadores adoptaban algún esquema metodológico, porque lo que más saltaba a la vista era la disparidad e irregularidad en su estructura entre cada una de ellas, lo que convirtió la revisión de sus capítulos en una actividad que involucraba al azar y a la buena suerte. Hallar dos tesis esquemáticamente semejantes, ya era un logro.

Las escuelas de postgrado de Derecho de las diferentes universidades exigen la persecución de un esquema (o enfoque) y, curiosamente, exclusiva y caprichosamente uno: el esquema científico-cuantitativo. Lo más probable es que en este momento en que usted lee este artículo algún proyecto de investigación de tesis o el informe final de tesis, en alguna Facultad de Derecho, esté siendo aprobada transgrediendo las normas que exigen el seguimiento de ese esquema, establecidas por esa misma Facultad.

Todas las tesis puesta bajo examen, fueron analizadas sin revelar a sus autores y los textos completos han sido registrados para posteriores investigaciones que intenten dar respuesta a preguntas que invaden fronteras filosóficas, en las que, como es sabido, no existe un consenso de arrebatarle el rótulo de ciencia a la práctica de indagación de los estudiosos del derecho, aunque conozcan las incompatibilidades entre los métodos doctrinarios y el método científico.

A pesar de que la lista de autores es amplia de quienes califican de científica la actividad intelectual ejecutada en las escuelas de derecho, no existen trabajos que intenten poner a prueba esta afirmación, teniendo en cuenta que esos mismos autores son los que han dejado en estanterías el producto de su investigación «científica» de grado (sí, sus tesis). Por lo que esta investigación está motivada en ese vacío, intencionado tal vez, que nos revela un fenómeno muy peculiar de entusiasmo, promovido por las Facultades de Derecho, que buscan la cientifización de la búsqueda de nuevos conocimientos en una disciplina que ha venido desarrollándose durante miles de años sin el apoyo metodológico de lo que hoy se denomina ciencia.

De aquí, me detuve a formular el problema de manera interrogativa, pues si todas las tesis que había observado hasta ese entonces pasaban por alto el empleo del método de la ciencia (entiéndase social), era posible que todas las tesis no aplicaran dicho método. Construí una pregunta de trabajo para plantearla de la siguiente manera: ¿Las tesis de postgrado de derecho han sido elaboradas empleando el método científico?

Para plantear la hipótesis, fue importante el contexto y algunos datos adicionales. Durante mi labor de asesor metodológico de investigaciones externo, cotidianamente noté no solo una contradicción entre el tesista y la institución que aprueba su tesis, sino una contradicción mucho más dérmica que se da entre las exigencias metódicas de la universidad y lo que los asesores internos -asignados al tesista por la misma universidad- consideran por investigación científica y que determinan el curso de las indagaciones. Noté que los asesores se desentienden completamente de las exigencias institucionales de plantear problemas de manera científica y de resolverlos de la misma manera, a saber, descuidando por completo 1) la explicitación y el establecimiento de relaciones entre las variables, 2) la determinación de cómo han de ser definidas operacionalmente, y 3) la especificación de cómo han de ser medidas para contrastar las afirmaciones propuestas. A consecuencia de esto, la hipótesis de trabajo se calibró para observar la presencia de dichas pautas del método de la ciencia que se aplican a esos estudios y contrastar lo siguiente: Las tesis de postgrado de derecho no emplean el método científico, al no justificar una relación válida entre sus variables, no definir operacionalmente a estas, ni aplicarles instrumentos matemáticos o estadísticos confiables y válidos para su medición. 
Los objetivos de esta investigación giraron claramente en indagar sobre esas pautas fijadas institucionalmente y la forma en cómo se aplicaron en las tesis de postgrado de derecho.

\section{Metodología}

Como unidad de análisis fue tomada la tesis de postgrado en Derecho culminada, presentada, sustentada y aprobada, y desde luego, puesta a disposición del público.

Las variables de esta investigación fueron las siguientes: la relación entre las variables en la tesis $(R)$, la operacionalización de esas variables en la tesis $(0)$, y los instrumentos de medición empleados para medir esas variables operacionalizadas en la tesis $(M)$.

La población consistió en las tesis de postgrado de la ciudad de Trujillo. Tomándose como muestra las tesis disponibles en estantería de los últimos diez años, entre el periodo 2004 al 2014. De todas las universidades de Trujillo, solo una universidad nacional y una privada fueron las únicas que contaron con tesis de postgrado accesibles al público en general, las otras no ofrecieron un catálogo de tesis, sea 1) porque no contaban con ninguna tesis o 2) porque no eran de acceso público al momento de solicitarlas; aunque lo que refirieron los administrativos de las bibliotecas es que en su mayoría se debió a la primera razón. Las universidades con tesis no accesibles al público no fueron consideradas en el estudio.

Las variables propuestas a medir en la presente investigación se constituyeron como ítems para su categorización y asignación de valores nominales a cada una de ellas de acuerdo a la escala de Likert, que son expuestas a continuación.

\section{Resultados}

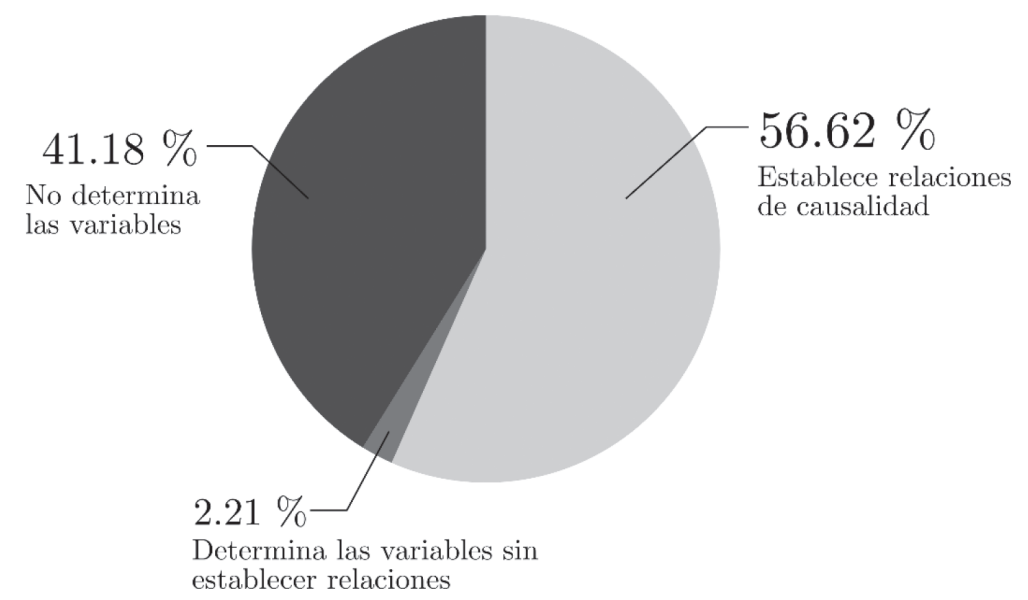

Gráfico No. 1

¿Qué relaciones se establecen entre las variables?

Cuadro N. ${ }^{\circ} 1$

\begin{tabular}{|c|c|c|c|c|c|}
\hline Categoría & $F$ & $\%$ & $\% A$ & $\begin{array}{l}\text { Estad. } \\
\text { Desc. }\end{array}$ & Valor \\
\hline No determina las variables & 56 & 41.18 & 41.18 & \multirow{5}{*}{$\begin{array}{l}\text { Media } \\
\text { E.E.M. } \\
\text { Moda } \\
\text { Des. Est. } \\
\text { Varianza } \\
\text { Curtosis } \\
\text { Asimetría } \\
\text { Mediana }\end{array}$} & \multirow{5}{*}{$\begin{array}{r}2.72 \\
0.13 \\
4.00 \\
1.47 \\
2.17 \\
-1.92 \\
-0.29\end{array}$} \\
\hline $\begin{array}{l}\text { Determina las variables sin establecer } \\
\text { relaciones }\end{array}$ & 3 & 2.21 & 43.38 & & \\
\hline Establece correlación entre las variables & 0 & 0.00 & 43.38 & & \\
\hline Establece relaciones de causalidad & 77 & 56.62 & 100.00 & & \\
\hline Total & 136 & 100.00 & & & \\
\hline
\end{tabular}


Al medir nuestra variable $R$, se encontró que en un $41.18 \%$ ( $f$ = 56) de las tesis el investigador no determina o establece expresamente las variables en su estudio, y un $2.21 \%$ las explicita, pero entre ellas no establece ningún tipo de relación. Sin embargo, todas las tesis que han establecido explícitamente las variables, que corresponde a un $56.62 \%$ $(f=77)$, establecen exclusivamente relación de causalidad, dejando de lado cualquier otro tipo de relación entre ellas, como es el caso de la correlación. No hay otro tipo de relación que se establezca entre las variables sino exclusivamente las de causa-efecto.

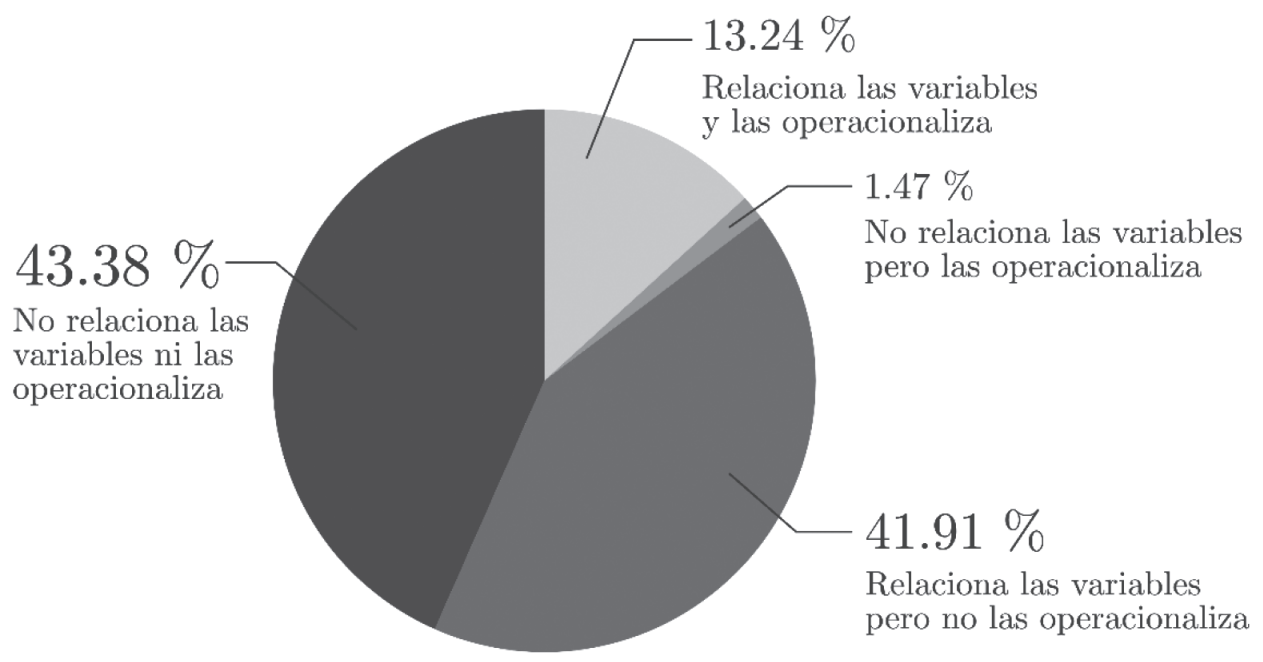

Gráfico N$^{\circ} 2$

¿Se operacionalizan las variables?

Cuadro N. ${ }^{\circ} 2$

\begin{tabular}{|c|c|c|c|c|c|}
\hline Categoría & $F$ & $\%$ & $\% A$ & $\begin{array}{l}\text { Estad. } \\
\text { Desc. }\end{array}$ & Valor \\
\hline $\begin{array}{l}\text { No relaciona las variables ni las } \\
\text { operacionaliza }\end{array}$ & 59 & 43.38 & 43.38 & \multirow{5}{*}{$\begin{array}{l}\text { Media } \\
\text { E.E.M. } \\
\text { Moda } \\
\text { Des. Est. } \\
\text { Varianza } \\
\text { Curtosis } \\
\text { Asimetría } \\
\text { Mediana }\end{array}$} & \multirow{5}{*}{$\begin{array}{r}1.85 \\
0.08 \\
1.00 \\
0.98 \\
0.96 \\
0.47 \\
1.18 \\
2\end{array}$} \\
\hline $\begin{array}{l}\text { Relaciona las variables pero no las } \\
\text { operacionaliza }\end{array}$ & 57 & 41.91 & 85.29 & & \\
\hline $\begin{array}{l}\text { No relaciona las variables pero las } \\
\text { operacionaliza }\end{array}$ & 2 & 1.47 & 86.76 & & \\
\hline Relaciona variables y las operacionaliza & 18 & 13.24 & 100.00 & & \\
\hline Total & 136 & 100.00 & & & \\
\hline
\end{tabular}

En estrecha vinculación con la variable $R$, está la variable $O$. En esta última se halló que un $43.38 \%(f=59)$ no explicita las variables, lo que lleva a suponer que tampoco las definen operacionalmente, debido a que no pueden definir algo que no existe como parte de la investigación. Por otro lado, el $41.91 \%(f=57)$, al establecer las relaciones de causalidad entre las variables, no las definen operacionalmente. Un número bastante reducido que constituye el $1.47 \%(f=2)$ del total de las tesis, al establecer explícitamente las variables sin especificar ninguna relación entre ellas, ofrece definiciones operacionales en su investigación. Por último, un $13.24 \%(f=18)$ que establecen relaciones de causalidad entre variables sí las definen operacionalmente. 
Gráfico N. ${ }^{\circ} 3$

¿Utilizan instrumentos de medición?

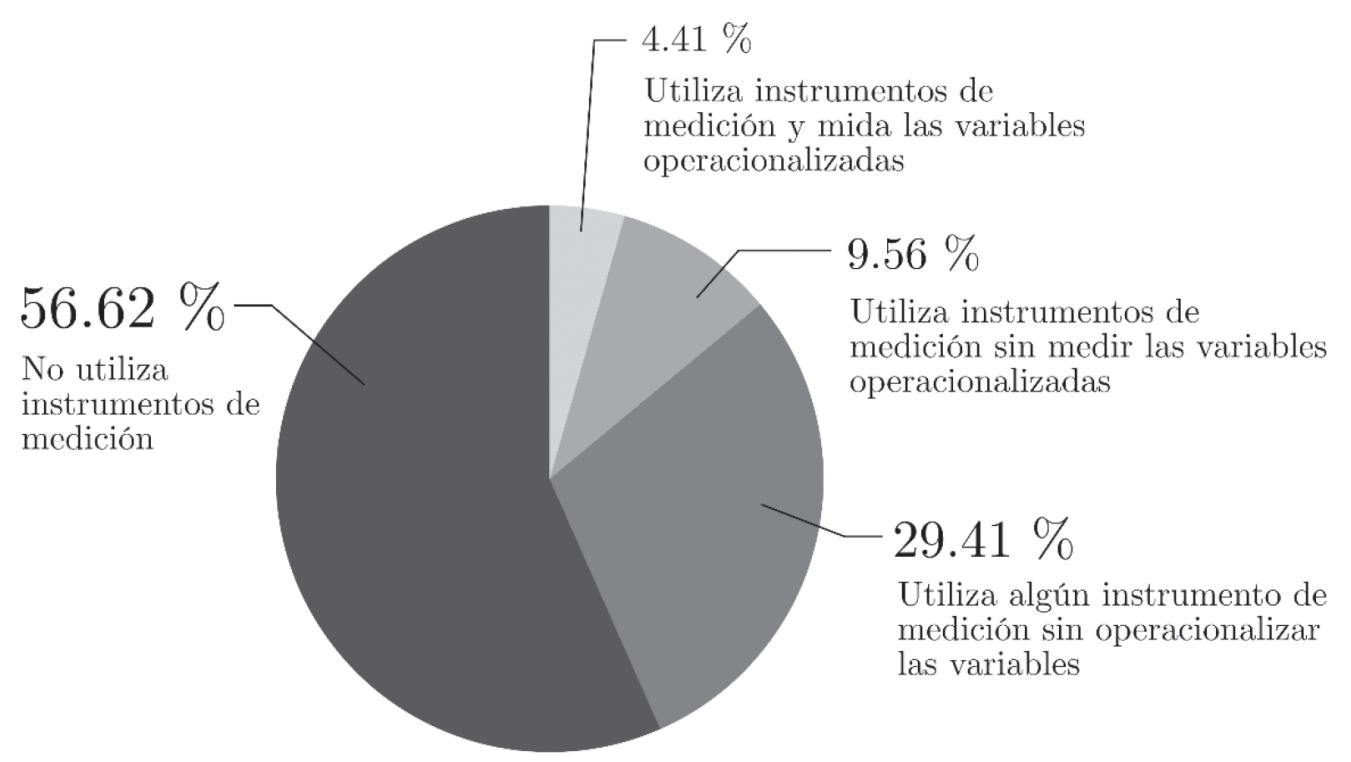

Cuadro N. ${ }^{\circ} 3$

\begin{tabular}{|c|c|c|c|c|c|}
\hline Categoría & $f$ & $\%$ & $\% A$ & $\begin{array}{l}\text { Estad. } \\
\text { Desc. }\end{array}$ & Valor \\
\hline No utiliza instrumentos de medición & 77 & 56.62 & 56.62 & \multirow{5}{*}{$\begin{array}{l}\text { Media } \\
\text { E.E.M. } \\
\text { Moda } \\
\text { Des. Est. } \\
\text { Varianza } \\
\text { Curtosis } \\
\text { Asimetría } \\
\text { Mediana }\end{array}$} & \multirow{5}{*}{$\begin{array}{r}1.62 \\
0.07 \\
1.00 \\
0.83 \\
0.70 \\
0.96 \\
1.29 \\
1\end{array}$} \\
\hline \multirow[t]{2}{*}{$\begin{array}{l}\text { Utiliza algún instrumento de medición sin } \\
\text { operacionalizar las variables }\end{array}$} & 40 & 29.41 & 86.03 & & \\
\hline & 13 & 9.56 & 95.59 & & \\
\hline $\begin{array}{l}\text { Utiliza instrumentos de medición y mide } \\
\text { las variables operacionalizadas }\end{array}$ & 6 & 4.41 & 100.00 & & \\
\hline Total & 136 & 100.00 & & & \\
\hline
\end{tabular}

En la variable $M$ se muestran los datos más importantes en lo que respecta al método científico. El $56.62 \%(f=$ 77) de los casos analizados no utilizan instrumentos de medición, que puede ser consecuencia natural de la inicial dificultad en la determinación de las variables como se observa del gráfico N. ${ }^{\circ} 1$. Un 29.41 $\%(f=40)$ utiliza instrumentos de medición sin antes haber definido operacionalmente las variables. Otro grupo que constituye el $9.56 \%(f=13)$, a pesar de haber realizado tal definición, utilizan instrumentos que no están dirigidos a su medición, midiendo variables no introducidas ni definidas en la tesis. Y, significativamente, del total de las tesis, sólo el $4.41 \%(f=6)$ utilizan instrumentos de medición para poner a prueba sus hipótesis.

\section{Discusión}

Puede sorprender que la exigencia en las investigaciones por parte de las escuelas de postgrado de Derecho consista en la adecuación a un formato de indagación cuantitativa, cuando ese formato es dejado de lado en casi la totalidad de ellas. Es interesante observar que las mismas hayan sido aprobadas por un comité evaluador previo a su sustentación y hayan dejado pasar por muy en alto la búsqueda metódicamente parametrada del tesista. La miopía del evaluador previo para verificar si se ajusta al método científico, se consuma en la sustentación 
y aprobación del trabajo por parte del jurado calificador. Es decir, tres personas (el jurado) conocedoras del enfoque cuantitativista del método que debió seguir el tesista lo dejan completamente de lado.

Debemos saber que en la metodología científica moderna para las ciencias sociales, se encuentran enfoques distintos y complementarios: enfoque cuantitativo y enfoque cualitativo, que son paradigmas de la investigación científica, pues ambos emplean procesos cuidadosos, sistemáticos y empíricos en su esfuerzo por generar conocimiento. Con el enfoque cuantitativo se usa la recolección de datos para probar hipótesis, con base en la medición numérica y el análisis estadístico, para establecer patrones de comportamiento y probar teorías, y el enfoque cualitativo se diferencia por la utilización y recolección de datos sin medición numérica para descubrir o afinar preguntas de investigación en el proceso de interpretación (Hernández, Fernández-Collado, Baptista, 2006).

Lo que permite que este fenómeno tan particular de elaboración de «trabajos científicos» en el Derecho, con el incumplimiento de reglas metódicas preestablecidas, y con la aceptación tácita institucionalizada de ese incumplimiento, es la confusión entre las ciencias sociales y el estudio doctrinario del derecho, manifestado en la naturaleza del objeto de estudio y el fin que persigue su investigación.

En ambos enfoques mencionados, adoptados en las ciencias sociales, se parte siempre de un hecho directa o indirectamente observable. En las ciencias sociales los hechos involucran comportamientos colectivos y la medición o internamiento participante en esos hechos es realizada para alcanzar el descubrimiento u obtener patrones recurrentes, lo que se repite de manera regular, y comprobar si estos datos una vez ordenados corresponden o no con las teorías que sustentan previamente la investigación. Sin embargo, el objeto de estudio en el estudio doctrinario del derecho, es la normatividad (Torres, 2006), el acceso a la experiencia jurídica para un jurista se efectúa a partir de la norma.

Parece que esta limitación voluntaria y epistemológicamente justificable de elegir a las normas como objeto de estudio, (sea o no una dimensión ligada a otras dos: hecho y valor), produce consecuentemente un alejamiento con la metodología del estudio de los fenómenos sociales fácticamente manifestados.

Esto no es nada nuevo, se conoce desde los antiguos griegos los diferentes niveles de la realidad, y la incompatibilidad o incorrespondencia entre los niveles fácticos (hechos) y lingüísticos (normas); pero, lo que sí resulta algo novedoso es que los estudiosos doctrinarios del derecho intenten construir argumentos de defensa que disimulen esta incompatibilidad interponiendo al método de las ciencias, afirmando muchas veces y de diferentes maneras, como lo hace Torres (2006), que la normatividad indefectiblemente conduce al conocimiento de las conductas humanas, en tanto constituyen su contenido sociológico-existencial, y a su valoración objetivada en la norma.

La idea de la cientifización de la doctrina jurídica, como un sano sentimiento de celos ante el avance paulatino pero seguro de las ciencias sociales, los impulsa y los obliga, como demuestra este trabajo, a adoptar formalmente un enfoque metodológico cuantitativo, aunque materialmente los doctrinarios sigan desarrollando «teorías» como hace miles de años atrás, persiguiendo incansablemente ideales de justicia (ideales), guiados por la prudencia (razón) y acompañados siempre en su razonamiento de los valores más importantes de los pueblos (valores).

Una muestra clara de lo último es lo que Fernández (2001) afirma sobre el estudio del Derecho, respecto a que éste no debería permanecer indiferente ante el acontecer social, desde que su misión es comprenderlo a fin de regularlo valorativamente otorgándole un sentido, y los resultados cognoscitivos y prácticos de su estudio, a decir de Torres (2006), pretender dar respuesta a una serie de situaciones sociales: conflictos, necesidades, intereses, acuerdos, entre otros.

Los resultados de este estudio vívido, impregnado de juicios valorativos, se agrupan y se interrelacionan sistemáticamente, como teorías, pero no como las que se desarrollan en las ciencias fácticas, no como teorías descriptivas o 
explicativas, sino como teorías prescriptivas. Como lo llama Bix (2006), estas teorías son elaboradas con un propósito configurador, constituyen una reconstrucción racional:

La «reconstrucción racional» con frecuencia juega un papel importante en la enseñanza del Derecho y el entrenamiento de los abogados. Aquí, de todos modos, debería notarse que la «reconstrucción racional» usada en la enseñanza puede diferenciarse poco, pero significativamente, comparada con la ofertada para fines puramente académicos. Un abogado debe tener buen ojo no sólo para la mejor reconstrucción de un área doctrinal confusa, sino también para la reconstrucción que parecerá mejor a los jueces con los cuales este abogado debe enfrentarse realmente. Así, si la mejor reconstrucción de los casos anteriores puede justificar algún derecho constitucional, pero los miembros actuales de la Corte Suprema del país son remisos a reconocer ese derecho, el abogado debe ser entrenado en una reconstrucción que excluya ese derecho (por lo menos hasta que cambien los miembros de la dicha corte) (Bix, 2006).

La doctrina frente a la normatividad realiza una actividad muy importante. La norma con su contenido general no determina la particularidad de cada caso; a la vez es dada aparentemente de forma aislada, siguiendo a principios que inicialmente no se le vinculan, y es formulada muchas veces en términos confusos o ambiguos, por lo que tiene que realizar funciones.
Sin el apoyo de la doctrina, no podríamos entender plenamente qué manda el texto de la norma, pues el significado de muchos términos ( $p$. ej. pretensión, apelación, debido proceso) sólo se comprende a cabalidad luego del estudio de textos doctrinales especializados. Estas funciones son parte esencial del sistema jurídico dentro de la familia roma-germánica, y que si bien la jurisprudencia puede eventualmente cumplir este papel, nunca llega a sustituir a la fuente doctrinal (Rubio, 2009).

La doctrina cumple funciones importantísimas en la comprensión del derecho, lo ha hecho desde siempre sin necesidad de la metodología científica. Ahora, a través de estos resultados, es evidente que las Facultades de Derecho pretenden adoptar métodos de investigación incompatibles con su objeto de estudio.

A pesar que en los círculos académicos doctrinarios es bastante conocida la diferencia entre lo que una norma -principio, sentencia, contrato- establece (de los que se derivan estudios jurídico-dogmáticos) y el comportamiento regulado por esa norma (de los que se derivan estudios empíricos) parece que el entusiasmo de cientifización olvidara y tratara de borrar levantando mucho polvo metodológico, dejando intactas las fronteras preclaras del nivel norma y del nivel hecho. La prueba indefectible de que el investigador se estrella contra los límites disimulados por la polvareda se exhibe públicamente en sus investigaciones.

\section{Conclusiones}

1. Con respecto a la determinación de variables, es significativa la cantidad de tesis que no las determinan $(41.18 \%)$. En la totalidad de las tesis, cuando se trata de establecer relaciones entre las variables prefieren exclusivamente las de causa y efecto (56.62\%) (variable independiente y variable dependiente) y únicamente entre dos variables. No se encuentran estudios correlacionales ni multivariados.

2. Las inexistencia de definiciones operacionales de las variables en las tesis $(43.38$ $\%)$, se encuentra necesariamente vinculada al gran número de tesis que no determinan sus variables. Sin embargo, en cuanto a la inexistencia de operacionalización en las tesis que establecen previamente relaciones de causalidad (41.91\%), no se encuentra evidencia fáctica observable que nos permita inferir la razón del por qué los investigadores prescinden de esta operacionalización. De esta manera, damos cuenta que el $85.29 \%$ de las tesis no definen operacionalmente sus variables. Por otro lado, solo un número reducido de tesis (13.24 \%) que establecen relaciones de causalidad sí lo hacen.

3. Los resultados más interesantes fueron los que se obtuvieron al observar los instrumentos de medición o su ausencia. Del inmenso pastel de dona, las más 
grandes tajadas pertenecen a los casos que no utilizan instrumentos de medición (56.62\%), y a los casos que utilizan instrumentos de medición sin haber definido operacionalmente las variables $(29.41 \%)$. Aunque se pudo observar intentos de medición, algunas tesis que sí definieron operacionalmente tropezaron con dificultades cuyas causas tampoco podían extraerse del análisis de las mismas tesis, midiendo variables ajenas a las previamente definidas (9.56\%). El pequeño número de tesis que utilizan instrumentos de medición para intentar poner a prueba sus hipótesis $(4.41 \%)$ demuestra la deficiente sujeción a las pautas más básicas del esquema cuantitativo de investigación científica.

\section{REFERENCIAS BIBLIOGRÁFICAS}

Bix, B. (2006). Teoría del derecho: Ambición y límites. Barcelona: Marcial Pons.

Fernández Sessarego, C. (2001). Derecho y persona: Introducción a la teoría del derecho. (4. ${ }^{\mathrm{a} e d .)}$ Lima: Grijley.
Hernández Sampieri, R; Fernández-Collado, C y Baptista Lucio, P. (2006). Metodología de la investigación. (4. ${ }^{a}$ ed.) México D.F.: McGraw-Hill.

Rubio Correa, M. (2009). El sistema jurídico: Introducción al derecho. (10. ${ }^{a}$ ed.) Lima: Pontificia

Universidad Católica del Perú.

Torres Vásquez, A. (2006). Introducción al derecho: Teoría General del Derecho. (3. ${ }^{\text {a }}$ ed.) Lima: Idemsa. 